\title{
Generalized Fokker-Planck equation: Derivation and exact solutions
}

\author{
S.I. Denisov ${ }^{1,2, a}$, W. Horsthemke ${ }^{3}$, and P. Hänggi ${ }^{4}$ \\ 1 Max-Planck-Institut für Physik komplexer Systeme, Nöthnitzer Straße 38, 01187 Dresden, Germany \\ 2 Sumy State University, 2 Rimsky-Korsakov Street, 40007 Sumy, Ukraine \\ 3 Department of Chemistry, Southern Methodist University, Dallas, Texas 75275-0314, USA \\ 4 Institut für Physik, Universität Augsburg, Universitätsstraße 1, 86135 Augsburg, Germany
}

Received 30 December 2008

Published online 8 April 2009 - (C) EDP Sciences, Società Italiana di Fisica, Springer-Verlag 2009

\begin{abstract}
We derive the generalized Fokker-Planck equation associated with the Langevin equation (in the Ito sense) for an overdamped particle in an external potential driven by multiplicative noise with an arbitrary distribution of the increments of the noise generating process. We explicitly consider this equation for various specific types of noises, including Poisson white noise and Lévy stable noise, and show that it reproduces all Fokker-Planck equations that are known for these noises. Exact analytical, time-dependent and stationary solutions of the generalized Fokker-Planck equation are derived and analyzed in detail for the cases of a linear, a quadratic, and a tailored potential.
\end{abstract}

PACS. 05.40.-a Fluctuation phenomena, random processes, noise, and Brownian motion - 05.10.Gg Stochastic analysis methods - 02.50.-r Probability theory, stochastic processes, and statistics

\section{Introduction}

Introduced just 100 years ago [1], the Langevin equation has become one of the most important and powerful tools for studying noise phenomena in systems coupled to a fluctuating environment [2]. The main advantage of this equation is that it provides a physically transparent and mathematically tractable description of the stochastic dynamics of such systems. The Langevin approach is especially effective if the noise that describes the action of the environment on the system can be represented as a time derivative, in the sense of generalized functions, of a stationary process with independent increments on non-overlapping intervals. In this case the solutions of the Langevin equation belong to the class of Markov processes whose properties are well known (see, e.g., Refs. [3-6]). The stationary processes with independent increments and zero initial state constitute a class of Lévy processes [7]. For brevity, we call the Lévy process, whose derivative produces a given noise, the noise generating process.

A large variety of physical, biological, financial and other systems is successfully described by the overdamped Langevin equation, i.e., the first-order stochastic differential equation. One of the main statistical characteristics of these systems is the probability density of the solution of this Langevin equation. If the noise results from the noise generating process, then the solution possesses the

\footnotetext{
a e-mail: stdenis@pks.mpg.de
}

Markovian property and the probability density satisfies a closed equation. Sometimes this equation is called the differential Chapman-Kolmogorov equation [8]. On the other hand, it is referred to as the Fokker-Planck equation for some particular cases. Specifically, a Gaussian distribution of the increments of the noise generating process corresponds to the ordinary Fokker-Planck equation [4-6], and heavy-tailed stable distributions to the fractional Fokker-Planck equation [9-14]. In order to capture these important cases, we call the closed equation for the probability density that corresponds to an arbitrary distribution of the increments the generalized Fokker-Planck equation. We have shown recently [15] that for additive noise the generalized Fokker-Planck equation can be represented in a unified way through the characteristic function of the noise generating process. Since the form and coefficients of this equation depend fundamentally on the distribution of the increments of the noise generating process, it provides a useful tool for studying the effects of different noises.

The aim of this paper is twofold. The first is to derive the generalized Fokker-Planck equation that corresponds to the overdamped Langevin equation driven by multiplicative noise with an arbitrary distribution of the increments of the noise generating process. The second is to solve this equation for specific cases and, on this basis, to study the distinctive effects of different noises on the system. 
The paper is organized as follows. In Section 2 we discuss the overdamped Langevin equation driven by multiplicative noise resulting from the noise generating process. In Section 3 we derive the generalized Fokker-Planck equation associated with this Langevin equation in terms of the transition probability density and characteristic function of the noise generating process. To confirm the validity of this equation, we consider in Section 4 various noises, including Poisson white and Lévy stable noises, for which the corresponding generalized Fokker-Planck equations are known. Several exact solutions of the generalized Fokker-Planck equation are derived in Section 5. In Section 6 we summarize our results.

\section{Overdamped Langevin equation}

The temporal evolution of the relevant degrees of freedom of dynamical systems that interact with a fluctuating environment is often described by the (dimensionless) overdamped Langevin equation

$$
\dot{x}(t)=f(x(t), t)+g(x(t), t) \xi(t) .
$$

For different systems the variables in this equation have different meanings, but to be concrete we will call $x(t)$ $[x(0)=0]$ a particle coordinate, $f(x, t)=-\partial U(x, t) / \partial x$ a force field, $U(x, t)$ an external deterministic potential, $\xi(t)$ a random force (noise) resulting from a fluctuating environment, and $g(x, t)$ a multiplicative noise term. Under certain conditions (see, e.g., Refs. [4,16]) the 'real' noise $\xi(t)$ with finite correlation time can be approximated by an idealized noise that effectively captures all the essential noise effects and turns $x(t)$ into a Markov process, with the result that many of these effects can be described analytically.

Because of the singular character of the idealized noise, equation (1) has to be interpreted with care. The starting point relies on the fact [17] that this noise is the time derivative, in the sense of generalized functions, of the noise generating process $\eta(t)$. According to this, the increment $\delta \eta(t)=\eta(t+\tau)-\eta(t)$ of $\eta(t)$ is defined as the time integral, $\delta \eta(t)=\int_{t}^{t+\tau} d t^{\prime} \xi\left(t^{\prime}\right)$, in the sense of convergence in distribution. Therefore, the increment $\delta x(t)=x(t+\tau)-x(t)$ of the particle coordinate during a time interval $\tau(\tau \rightarrow 0)$ can be written in the form

$$
\delta x(t)=f(x(t), t) \tau+g(x(t), t) \delta \eta(t),
$$

which defines the meaning of equation (1) in the Ito interpretation [18] (see also Refs. [2,4-6]).

For a fixed $\tau$, the distribution of the increments $\delta \eta(j \tau)$ $(j=0,1,2, \ldots)$ is completely described by the transition probability density $p\left(\eta_{j+1}, \tau \mid \eta_{j}\right)$, where $\eta_{j+1}$ and $\eta_{j}$ denote the possible values of $\eta(j \tau+\tau)$ and $\eta(j \tau)$, respectively. These densities are properly normalized, $\int_{-\infty}^{\infty} d \eta_{j+1}$ $\times p\left(\eta_{j+1}, \tau \mid \eta_{j}\right)=1$, and satisfy the following condition $\lim _{\tau \rightarrow 0} p\left(\eta_{j+1}, \tau \mid \eta_{j}\right)=\delta(\Delta \eta)$, where $\delta(\cdot)$ stands for the Dirac $\delta$ function and $\Delta \eta=\eta_{j+1}-\eta_{j}$. Moreover, if the first moment of $\eta(j \tau+\tau)$ exists, it is assumed to be zero, i.e., $\int_{-\infty}^{\infty} d \eta_{j+1} \eta_{j+1} p\left(\eta_{j+1}, \tau \mid \eta_{j}\right)=0$.

The noise generating process, i.e., the stationary Markov process $\eta(t)=\lim _{\tau \rightarrow 0} \sum_{j=0}^{[t / \tau]-1} \delta \eta(j \tau)$ with $\eta(0)=0$ and $[t / \tau]$ denoting the integer part of $t / \tau$, is also completely defined by the transition probability density $p\left(\eta_{j+1}, \tau \mid \eta_{j}\right)$. We note in this regard that all transition probability densities of the form $p\left(\eta_{j+l}, l \tau \mid \eta_{j}\right) \quad(l=2,3, \ldots)$ can be expressed through $p\left(\eta_{j+1}, \tau \mid \eta_{j}\right)$ by using the ChapmanKolmogorov equation [3-5]. In particular, for $l=2$ it yields $p\left(\eta_{j+2}, 2 \tau \mid \eta_{j}\right)=\int_{-\infty}^{\infty} d \eta_{j+1} p\left(\eta_{j+2}, \tau \mid \eta_{j+1}\right) p\left(\eta_{j+1}\right.$, $\left.\tau \mid \eta_{j}\right)$. Thus, the statistical properties of solutions of the Langevin equation (1) can be characterized by $p\left(\eta_{j+1}, \tau \mid \eta_{j}\right)$ as well. Next, for simplicity, we additionally assume that $p\left(\eta_{j+1}, \tau \mid \eta_{j}\right)=p(\Delta \eta, \tau)$.

If, for example, the transition probability density is Gaussian, i.e.,

$$
p(\Delta \eta, \tau)=\frac{e^{-\Delta \eta^{2} /(4 D \tau)}}{\sqrt{4 \pi D \tau}},
$$

then $\eta(n \tau)=\sum_{j=0}^{n-1} \delta \eta(j \tau)$ is a discrete-time Wiener process, which is fully characterized by two parameters,

$$
\langle\delta \eta(j \tau)\rangle=0, \quad\langle\delta \eta(j \tau) \delta \eta(l \tau)\rangle=2 D \delta_{j l} \tau .
$$

The angular brackets denote averaging over the increments $\delta \eta(j \tau)$, and $\delta_{n m}$ is the Kronecker symbol. These formulas are the discrete-time versions of the mean $\langle\xi(t)\rangle=0$ and the correlation function $\left\langle\xi(t) \xi\left(t^{\prime}\right)\right\rangle=2 D \delta\left(t-t^{\prime}\right)$ of a Gaussian white noise $\xi(t)$ of intensity $D$. Thus equations (2) and (4) completely specify the Langevin equation (1) driven by multiplicative Gaussian white noise [4].

To derive the Fokker-Planck equation, it is typically assumed that the first two moments of $\delta \eta(j \tau)$ exist $[3,6]$. However, if $p(\Delta \eta, \tau)$ is a heavy-tailed function of $\Delta \eta$, then the second moment does not exist. In this case, the derivation of the Fokker-Planck equation that corresponds to the Langevin equation (1) must be based solely on equation (2). We emphasize that noises characterized by heavytailed transition probability densities $p(\Delta \eta, \tau)$ differ qualitatively from those characterized by $p(\Delta \eta, \tau)$ with finite variances. Specifically, the latter have a frequency independent power spectral density $\int_{-\infty}^{\infty} d t e^{-i \omega t}\langle\xi(0) \xi(t)\rangle(\omega$ is the frequency) and consequently they are called white noises. In contrast, the power spectrum of the former does not exist. Nevertheless, they are a very useful tool for studying an important class of random processes that exhibit rare but large jumps.

\section{Generalized Fokker-Planck equation}

We define the probability density of the particle coordinate $x(t)$ in the usual way:

$$
P(x, t)=\langle\delta(x-x(t))\rangle
$$


To derive the evolution equation for this probability density, we need to be able to express the average values of $F(x(t))$ and $F(x(t), \delta \eta(t))$ in terms of $P(x, t)$; the functions $F(x)$ and $F(x, y)$ are assumed to be deterministic. Keeping in mind the above definition of averaging, $\langle F(x(t))\rangle$ means averaging $F(x(t))$ over all increments $\delta \eta(j \tau)$ with $j=0,1, \ldots,[t / \tau]-1$ and $\tau \rightarrow 0$. It is obvious from equations (2) and (5) that the result can be represented as an average with respect to the distribution of $x(t)$, i.e.,

$$
\langle F(x(t))\rangle=\int_{-\infty}^{\infty} d x F(x) P(x, t) .
$$

In order to express $\langle F(x(t), \delta \eta(t))\rangle$ in terms of $P(x, t)$, we use a two-stage averaging procedure [19]. Since the variables $x(t)$ and $\delta \eta(t)$ are statistically independent and distributed according to the probability densities $P(x, t)$ and $p(\Delta \eta, \tau)$, respectively, we readily obtain

$$
\langle F(x(t), \delta \eta(t))\rangle=\int_{-\infty}^{\infty} d x P(x, t) \int_{-\infty}^{\infty} d y F(x, y) p(y, \tau) .
$$

To proceed, we introduce the Fourier transform, $P_{k}(t)$, of $P(x, t)$ according to the formula

$$
\mathcal{F}\{u(x)\} \equiv u_{k}=\int_{-\infty}^{\infty} d x e^{-i k x} u(x),
$$

and find, using definition (5), that $P_{k}(t)=\left\langle e^{-i k x(t)}\right\rangle$. Equation (2) implies that for $\tau \rightarrow 0$ the increment of $P_{k}(t)$, i.e., $\delta P_{k}=P_{k}(t+\tau)-P_{k}(t)$, can be written in the form

$$
\begin{aligned}
\delta P_{k}= & -i k \tau\left\langle e^{-i k x(t)} f(x(t), t)\right\rangle \\
& +\left\langle e^{-i k x(t)}\left(e^{-i k g(x(t), t) \delta \eta(t)}-1\right)\right\rangle .
\end{aligned}
$$

In accordance with (6) and (8), the first term on the righthand side of equation (9) reduces to

$$
i k\left\langle e^{-i k x(t)} f(x(t), t)\right\rangle=\mathcal{F}\left\{\frac{\partial}{\partial x} f(x, t) P(x, t)\right\},
$$

and equation (7) for the second term gives

$$
\begin{aligned}
& \left\langle e^{-i k x(t)}\left(e^{-i k g(x(t), t) \delta \eta(t)}-1\right)\right\rangle= \\
& \int_{-\infty}^{\infty} d y e^{-i k y}\left[p_{k g(y, t)}(\tau)-1\right] P(y, t),
\end{aligned}
$$

where $p_{k}(\tau)=\mathcal{F}\{p(x, \tau)\}=\left\langle e^{-i k \delta \eta(t)}\right\rangle$ is the characteristic function of $\delta \eta(t)$.

Substituting (10) and (11) into equation (9), dividing it by $\tau$ and taking the limit $\tau \rightarrow 0$, we obtain the following equation:

$$
\begin{aligned}
\frac{\partial}{\partial t} P_{k}(t)= & -\mathcal{F}\left\{\frac{\partial}{\partial x} f(x, t) P(x, t)\right\} \\
& +\int_{-\infty}^{\infty} d y e^{-i k y} \phi_{k g(y, t)} P(y, t)
\end{aligned}
$$

with

$$
\phi_{k}=\lim _{\tau \rightarrow 0} \frac{1}{\tau}\left[p_{k}(\tau)-1\right] .
$$

Since the transition probability density $p(\Delta \eta, \tau)$ is normalized, i.e., $p_{0}(\tau)=1$, the limit (13) must satisfy the condition $\phi_{0}=0$. If $k \neq 0$, then there exist three different cases, depending on how quickly $p_{k}(\tau)-1$ tends to zero as $\tau \rightarrow 0$. First, if $p_{k}(\tau)-1=o(\tau)$, then $\phi_{k}=0$ and the noise does not effect the system at all. Second, if $p_{k}(\tau)-1$ tends to zero slower than $\tau$, then $\left|\phi_{k}\right|=\infty$, i.e., the influence of the noise is so strong that the system relaxes instantaneously to the final state. Finally, the case we are interested in corresponds to $p_{k}(\tau)-1=O(\tau)$, i.e., $0<\left|\phi_{\kappa}\right|<\infty$ and the noise acts on the system in a non-trivial way.

We apply the inverse Fourier transform, defined as

$$
\mathcal{F}^{-1}\left\{u_{k}\right\} \equiv u(x)=\frac{1}{2 \pi} \int_{-\infty}^{\infty} d k e^{i k x} u_{k},
$$

to equation (12). Using the definition (13), we obtain

$$
\mathcal{F}^{-1}\left\{e^{-i k y} \phi_{k g(y, t)}\right\}=\frac{1}{|g(y, t)|} \phi\left(\frac{x-y}{g(y, t)}\right),
$$

where the function

$$
\phi(x)=\lim _{\tau \rightarrow 0} \frac{1}{\tau}[p(x, \tau)-\delta(x)]
$$

is a special characteristic of $p(\Delta \eta, \tau)$ for $\tau \rightarrow 0$ that describes the influence of noise on the system. Therefore, the desired generalized Fokker-Planck equation that corresponds to the Langevin equation (1) driven by multiplicative noise, which results from an arbitrary noise generating process, takes the form

$$
\begin{aligned}
\frac{\partial}{\partial t} P(x, t)= & -\frac{\partial}{\partial x} f(x, t) P(x, t) \\
& +\int_{-\infty}^{\infty} d y \frac{P(y, t)}{|g(y, t)|} \phi\left(\frac{x-y}{g(y, t)}\right) .
\end{aligned}
$$

In accordance with the definition (5), the solution of this equation must be normalized and satisfy the initial condition $P(x, 0)=\delta(x)$.

To gain more insight into the connection between the generalized Fokker-Planck equation and the properties of the noise, we introduce the characteristic function $S_{k}=$ $\left\langle e^{-i k \eta(1)}\right\rangle$ of the noise generating process $\eta(t)$ at $t=1$. With the formula $\eta(1)=\lim _{\tau \rightarrow 0} \sum_{j=0}^{[1 / \tau]-1} \delta \eta(j \tau)$, it can be rewritten as $S_{k}=\lim _{\tau \rightarrow 0}\left(p_{k}(\tau)\right)^{[1 / \tau]}$. Then replacing $p_{k}(\tau)$ by $1+\tau \phi_{k}$ and taking into account that $\lim _{\varepsilon \rightarrow 0}(1+$ $\varepsilon)^{1 / \varepsilon}=e$, we find $S_{k}=e^{\phi_{k}}$, i.e., $\phi_{k}=\ln S_{k}$. Thus, from equation (12) we obtain an alternative representation of the generalized Fokker-Planck equation:

$$
\begin{aligned}
\frac{\partial}{\partial t} P(x, t)= & -\frac{\partial}{\partial x} f(x, t) P(x, t) \\
& +\mathcal{F}^{-1}\left\{\int_{-\infty}^{\infty} d y e^{-i k y} P(y, t) \ln S_{k g(y, t)}\right\}
\end{aligned}
$$


In the particular case of additive noise, where $g(x, t)=1$, equation (12) becomes

$$
\frac{\partial}{\partial t} P_{k}(t)=-\mathcal{F}\left\{\frac{\partial}{\partial x} f(x, t) P(x, t)\right\}+P_{k}(t) \phi_{k},
$$

and the generalized Fokker-Planck equation (18) simplifies to the equation

$$
\frac{\partial}{\partial t} P(x, t)=-\frac{\partial}{\partial x} f(x, t) P(x, t)+\mathcal{F}^{-1}\left\{P_{k}(t) \ln S_{k}\right\},
$$

which was derived in reference [15].

We note that the problem of deriving the generalized Fokker-Planck equation that corresponds to the Langevin equation (1) has been considered earlier in terms of the Lévy measure of the noise generating process [20-23]. In contrast, the generalized Fokker-Planck equations (18) and (20) are derived here in terms of the characteristic function $S_{k}$ of this process at $t=1$. Since the stationary processes with independent increments are infinitely divisible, $\ln S_{k}$ can be represented by the Lévy-Khintchine formula that connects $S_{k}$ with the Lévy measure [7]. Hence, both approaches are, in fact, equivalent and lead to different forms of the generalized Fokker-Planck (see also Sect. 4.5). It seems, however, that the above approach which deals with the transition probability density of the noise generating process is more convenient for applications. Indeed, the transition probability density completely describes the noise generating process and, in accordance with (13) and $S_{k}=e^{\phi_{k}}$, explicitly represents the characteristic function $S_{k}$. At the same time, there is no simple way to invert the Lévy-Khintchine formula, i.e., to express the Lévy measure through $S_{k}[24]$.

\section{Special cases of the generalized Fokker-Planck equation}

In order to confirm the validity of equations (17), (18) and (20) and to demonstrate their usefulness, we consider several specific noises for which the corresponding Fokker-Planck equations associated with the Langevin equation (1) are already known.

\subsection{Gaussian white noise}

The transition probability density $p(\Delta \eta, \tau)$ for Gaussian white noise is given by formula (3). Accordingly, $p_{k}(\tau)=$ $e^{-D \tau k^{2}}, \phi_{k}=-D k^{2}$, and $S_{k}=e^{-D k^{2}}$. Then, taking into account that $\mathcal{F}^{-1}\left\{P_{k}(t) k^{2}\right\}=-\partial^{2} P(x, t) / \partial x^{2}$, we find that in the case of additive Gaussian white noise equation (20) reduces indeed to the ordinary Fokker-Planck equation $[4-6]$

$$
\frac{\partial}{\partial t} P(x, t)=-\frac{\partial}{\partial x} f(x, t) P(x, t)+D \frac{\partial^{2}}{\partial x^{2}} P(x, t)
$$

If the Gaussian white noise is multiplicative, then $\phi_{k g(y, t)}=-D k^{2} g^{2}(y, t)$ and

$$
\begin{aligned}
\mathcal{F}^{-1}\left\{\int_{-\infty}^{\infty} d y e^{-i k y} \phi_{k g(y, t)}\right. & P(y, t)\}= \\
-D \mathcal{F}^{-1}\left\{k^{2} \mathcal{F}\right. & \left.\left\{g^{2}(x, t) P(x, t)\right\}\right\} \\
& =D \frac{\partial^{2}}{\partial x^{2}} g^{2}(x, t) P(x, t) .
\end{aligned}
$$

Applying the inverse Fourier transform to equation (12) and using the above result, we again obtain the ordinary Fokker-Planck equation [4-6]

$$
\frac{\partial}{\partial t} P(x, t)=-\frac{\partial}{\partial x} f(x, t) P(x, t)+D \frac{\partial^{2}}{\partial x^{2}} g^{2}(x, t) P(x, t),
$$

which corresponds to the Ito interpretation of the Langevin equation (1) driven by multiplicative Gaussian white noise. We note that the last equation can also be derived from equation (17) with $\phi(\cdot)=D \partial^{2} \delta(\cdot) / \partial x^{2}$ for this case.

\subsection{Poisson white noise}

As a second example we consider Poisson white noise, i.e., a random sequence of $\delta$-pulses, defined as $[25,26]$

$$
\xi(t)=\sum_{i=1}^{n(t)} z_{i} \delta\left(t-t_{i}\right)
$$

Here $n(t)$ is a Poisson counting process with the probability $P(n(t)=n)=(\lambda t)^{n} e^{-\lambda t} / n$ ! of $n \geq 0$ arrivals in the interval $(0, t], \lambda$ is the rate of the process, $t_{i}$ are the (random) arrival times of this process, and $z_{i}$ are independent random variables of zero mean distributed with the same probability density $q(z)$. It is assumed also that $\xi(t)=0$ if $n(t)=0$. The noise generating process $\eta(t)$ is a step-wise constant Markov process whose increments $\delta \eta(t)=\int_{t}^{t+\tau} d t^{\prime} \xi\left(t^{\prime}\right)$ are given by

$$
\delta \eta(t)=\left\{\begin{array}{l}
0, \quad \text { if } n(\tau)=0, \\
\sum_{i=1}^{n(\tau)} z_{i}, \quad \text { if } n(\tau) \geq 1 .
\end{array}\right.
$$

In order to find the transition probability density $p(\Delta \eta, \tau)$, we use the definition $p(\Delta \eta, \tau)=\langle\delta(\Delta \eta-\delta \eta(t))\rangle$ which, together with (25), yields

$$
p(\Delta \eta, \tau)=P_{0}(\tau) \delta(\Delta \eta)+W(\Delta \eta, \tau) .
$$

The first term on the right-hand side of this formula is the probability density of $\delta \eta(t)$ under the condition that none of the $\delta$-pulses occurred during the time interval $\tau$. The second term,

$$
\begin{aligned}
W(\Delta \eta, \tau)= & \sum_{n=1}^{\infty} P_{n}(\tau) \int_{-\infty}^{\infty} \ldots \int_{-\infty}^{\infty} \delta\left(\Delta \eta-\sum_{i=1}^{n} z_{i}\right) \\
& \times \prod_{j=1}^{n} q\left(z_{j}\right) d z_{j},
\end{aligned}
$$


represents the probability density of $\delta \eta(t)$ under the condition that at least one pulse occurs during this time interval. Taking the probabilities $P_{n}(\tau)=P(n(\tau)=n)$ with linear accuracy in $\tau$, i.e., $P_{0}(\tau)=1-\lambda \tau, P_{1}(\tau)=\lambda \tau$, and $P_{n \geq 2}(\tau)=0$, we obtain from formulas (26) and (27)

$$
p(\Delta \eta, \tau)=(1-\lambda \tau) \delta(\Delta \eta)+\lambda \tau q(\Delta \eta) .
$$

In accordance with the definition (16), for this probability density $\phi(x)=\lambda[q(x)-\delta(x)]$, and the generalized FokkerPlanck equation (17) reads

$$
\begin{aligned}
\frac{\partial}{\partial t} P(x, t)= & -\frac{\partial}{\partial x} f(x, t) P(x, t)-\lambda P(x, t) \\
& +\lambda \int_{-\infty}^{\infty} d y \frac{P(y, t)}{|g(y, t)|} q\left(\frac{x-y}{g(y, t)}\right) .
\end{aligned}
$$

For $g(x, t)=1$, i.e., in the case of additive Poisson white noise, this equation is consistent with those reported previously [25-28]. Of course, since $S_{k}=e^{-\lambda\left(1-q_{k}\right)}$, $q(x)=\mathcal{F}^{-1}\left\{q_{k}\right\}$ and $\delta(x)=\mathcal{F}^{-1}\{1\}$, the same form of the equation $(29)$ (with $g(x, t)=1$ ) follows from equation (20) as well. We note that a wide class of white noises, which are represented by random sequences of $\delta$-pulses with a mean number $\lambda$ of pulses per unit time, is characterized by the same transition probability density (28). Therefore, the generalized Fokker-Planck equation (29) is also valid for all these noises.

\subsection{Compound noise}

Next we consider the noise $\xi(t)=\sum_{m=1}^{M} \xi_{m}(t)$ composed of a set of independent noises $\xi_{m}(t)$. In this case the noise generating process can be written in the form

$$
\eta(t)=\lim _{\tau \rightarrow 0} \sum_{m=1}^{M} \sum_{j=0}^{[t / \tau]-1} \delta \eta_{m}(j \tau)
$$

Because of the statistical independence of the increments $\delta \eta_{m}(j \tau)$ of the partial generating processes $\eta_{m}(t)$, the characteristic function $S_{k}=\left\langle e^{-i k \eta(1)}\right\rangle$ of $\eta(1)$ is expressed through the characteristic functions $S_{m k}=\left\langle e^{-i k \eta_{m}(1)}\right\rangle$ of $\eta_{m}(1)$ as follows: $S_{k}=\prod_{m=1}^{M} S_{m k}$. Therefore, in the case of additive compound noise the generalized Fokker-Planck equation (20) becomes

$$
\begin{aligned}
\frac{\partial}{\partial t} P(x, t)= & -\frac{\partial}{\partial x} f(x, t) P(x, t) \\
& +\sum_{m=1}^{M} \mathcal{F}^{-1}\left\{P_{k}(t) \ln S_{m k}\right\} .
\end{aligned}
$$

In particular, if $M=2$ and $\xi_{1}(t)$ and $\xi_{2}(t)$ are Gaussian and Poisson white noises, respectively, then equation (31) reduces to [8]

$$
\begin{aligned}
\frac{\partial}{\partial t} P(x, t)= & -\frac{\partial}{\partial x} f(x, t) P(x, t)+D \frac{\partial^{2}}{\partial x^{2}} P(x, t) \\
& -\lambda P(x, t)+\lambda \int_{-\infty}^{\infty} d y P(y, t) q(x-y) .
\end{aligned}
$$

\subsection{Lévy stable noise}

The generalized central limit theorem [29] implies that for a wide class of properly scaled transition probability densities $p(\Delta \eta, \tau)$, the characteristic function $S_{k}$ corresponds to Lévy stable distributions, $S_{k}=S_{k}(\alpha, \beta, \gamma, \rho)$. It is well known [30] that $S_{k}(\alpha, \beta, \gamma, \rho)$ depends on four parameters: an index of stability $\alpha \in(0,2]$, a skewness parameter $\beta \in[-1,1]$, a scale parameter $\gamma \in(0, \infty)$, and a location parameter $\rho \in(-\infty, \infty)$. Assuming in accordance with the initial condition $P(x, 0)=\delta(x)$ that $\rho=0$ and excluding from consideration the singular case when $\alpha=1$ and $\beta \neq 0$ simultaneously (in this case $\left|\phi_{k}\right|=\infty$ ), we obtain $S_{k}=S_{k}(\alpha, \beta, \gamma)$, where [30]

$$
S_{k}(\alpha, \beta, \gamma)=\exp \left[-\gamma|k|^{\alpha}\left(1+i \beta \operatorname{sgn}(k) \tan \frac{\pi \alpha}{2}\right)\right] .
$$

In the following we assume for simplicity that the condition $g(y, t)>0$ holds for all $y$ and $t$. In this case

$$
\ln S_{k g(y, t)}=g^{\alpha}(y, t) \ln S_{k}(\alpha, \beta, \gamma),
$$

and the generalized Fokker-Planck equation (18) becomes

$\frac{\partial}{\partial t} P(x, t)=-\frac{\partial}{\partial x} f(x, t) P(x, t)+\mathcal{F}^{-1}\left\{G_{k}(t) \ln S_{k}(\alpha, \beta, \gamma)\right\}$,

where

$$
G_{k}(t)=\mathcal{F}\left\{g^{\alpha}(x, t) P(x, t)\right\} .
$$

Equation (35) can be rewritten in a form containing the Riemann-Liouville derivatives defined as [31]

$$
{ }_{s} D_{ \pm}^{\sigma} h(x)=\frac{( \pm 1)^{n}}{\Gamma(n-\sigma)} \frac{d^{n}}{d x^{n}} \int_{0}^{s \pm x} d y h(x \mp y) y^{n-\sigma-1}
$$

where ${ }_{s} D_{+}^{\sigma}$ and ${ }_{s} D_{-}^{\sigma}$ denote the operators of the left- and right-hand side derivatives of the order $\sigma(0<\sigma<\infty)$, respectively. The function $h(x)$ is defined on the interval $[-s, s], n=1+[\sigma]$, and $\Gamma(z)$ is the Gamma function. Using the characteristic function (33), we first represent its natural logarithm as follows:

$$
\ln S_{k}(\alpha, \beta, \gamma)=-\gamma \frac{(1+\beta)(i k)^{\alpha}+(1-\beta)(-i k)^{\alpha}}{2 \cos (\pi \alpha / 2)} .
$$

Taking the Fourier transform of equation (37) with $h(x)=$ $g^{\alpha}(x, t) P(x, t)$, we find

$$
( \pm i k)^{\alpha} G_{k}(t)=\mathcal{F}\left\{{ }_{\infty} D_{ \pm}^{\alpha} g^{\alpha}(x, t) P(x, t)\right\},
$$

and combining this result with (38) we obtain the fractional Fokker-Planck equation

$$
\begin{aligned}
\frac{\partial}{\partial t} P(x, t)= & -\frac{\partial}{\partial x} f(x, t) P(x, t)-\frac{\gamma}{2 \cos (\pi \alpha / 2)}[(1+\beta) \\
& \left.\times_{\infty} D_{+}^{\alpha}+(1-\beta)_{\infty} D_{-}^{\alpha}\right] g^{\alpha}(x, t) P(x, t)
\end{aligned}
$$


Equation (40) reproduces all known forms of the fractional Fokker-Planck equation that corresponds to the Langevin equation (1) driven by Lévy stable noise. It can be easily rewritten in a form containing the Riesz derivative defined as $[31]$

$$
\frac{\partial^{\alpha}}{\partial|x|^{\alpha}} h(x)=-\mathcal{F}^{-1}\left\{|k|^{\alpha} h_{k}\right\} .
$$

With the help of this definition and the relations

$$
\left({ }_{\infty} D_{+}^{\alpha}+{ }_{\infty} D_{-}\right) h(x)=2 \cos \frac{\pi \alpha}{2} \mathcal{F}^{-1}\left\{|k|^{\alpha} h_{k}\right\}
$$

and

$$
\left({ }_{\infty} D_{+}^{\alpha}-_{\infty} D_{-}\right) h(x)=2 \sin \frac{\pi \alpha}{2} \frac{\partial}{\partial x} \mathcal{F}^{-1}\left\{|k|^{\alpha-1} h_{k}\right\}
$$

which follow directly from the Fourier representation ${ }_{\infty} D_{ \pm}^{\alpha} h(x)=\mathcal{F}^{-1}\left\{( \pm i k)^{\alpha} h_{k}\right\}$ of the Riemann-Liouville derivatives, equation (40) reduces to

$$
\begin{aligned}
\frac{\partial}{\partial t} P(x, t)= & -\frac{\partial}{\partial x} f(x, t) P(x, t)+\gamma \frac{\partial^{\alpha}}{\partial|x|^{\alpha}} g^{\alpha}(x, t) P(x, t) \\
& +\gamma \beta \tan \frac{\pi \alpha}{2} \frac{\partial}{\partial x} \frac{\partial^{\alpha-1}}{\partial|x|^{\alpha-1}} g^{\alpha}(x, t) P(x, t)
\end{aligned}
$$

It is obvious that for $\alpha=2$ this equation takes the form of the ordinary Fokker-Planck equation (23) with $D=\gamma$. For $\alpha<2$, various special cases of equation (44) have been given previously in references [9-14].

\subsection{Infinite divisibility of the noise generating process}

It is well known (see, e.g., Refs. $[7,24]$ ) that any stationary process with independent increments, including the noise generating process, is infinitely divisible. This means that the condition $S_{k}=\left(S_{k}^{(n)}\right)^{n}$ with $S_{k}^{(n)}$ being a characteristic function holds for each positive integer $n$. In this case, $\ln S_{k}$ can be represented in the form [24]

$$
\ln S_{k}=\int_{-\infty}^{\infty} d z \rho(z) \frac{e^{-i k z}-1+i k \sin z}{z^{2}}
$$

where $\rho(z)$ is the density of the Lévy measure of $\eta(1)$. Accordingly, with the integral representation $\delta(\cdot)=(1 / 2 \pi)$ $\times \int_{-\infty}^{\infty} d k e^{i k(\cdot)}$ of the $\delta$ function, the last term in equation (18) can be written as follows:

$$
\begin{aligned}
\mathcal{F}^{-1}\left\{\int_{-\infty}^{\infty} d y e^{-i k y} P(y, t) \ln S_{k g(y, t)}\right\}= \\
\quad \int_{-\infty}^{\infty} d z \frac{\rho(z)}{z^{2}} \int_{-\infty}^{\infty} d y(\delta[x-y-z g(y, t)] \\
\left.-\delta(x-y)+\sin z \frac{\partial}{\partial x} g(x, t) \delta(x-y)\right) P(y, t) .
\end{aligned}
$$

Evaluating the integral over $y$ and using the formula

$$
\begin{aligned}
\int_{-\infty}^{\infty} & d y \delta[x-y-z g(y, t)] P(y, t)=\sum_{n=0}^{\infty}(-z)^{n} \\
\times & \frac{\partial^{n}}{\partial x^{n}} g^{n}(x, t) P(x, t) \equiv \exp \left(-z \frac{\partial}{\partial x} g(x, t)\right) P(x, t),
\end{aligned}
$$

which follows from the Taylor expansion $\delta[x-y-$ $z g(y, t)]=\sum_{n=0}^{\infty}(-1)^{n} g^{n}(y, t)(\partial / \partial x)^{n} \delta(x-y)$, we obtain the generalized Fokker-Planck equation

$$
\begin{aligned}
\frac{\partial}{\partial t} P(x, t)= & -\frac{\partial}{\partial x} f(x, t) P(x, t) \\
& +\int_{-\infty}^{\infty} d z \frac{\rho(z)}{z^{2}}\left[\exp \left(-z \frac{\partial}{\partial x} g(x, t)\right)\right. \\
& \left.-1+\sin z \frac{\partial}{\partial x} g(x, t)\right] P(x, t),
\end{aligned}
$$

which was derived in reference [22] using a functional approach.

\section{Exact solutions of the generalized Fokker-Planck equation}

An important aspect of the generalized Fokker-Planck equation (20), which corresponds to the Langevin equation (1) driven by additive noise, is that in some cases it can be solved for all characteristic functions $S_{k}$, i.e., for all noises represented by a time derivative of the noise generating process. This provides a unique opportunity to study in detail the effect of different noises on the same system. In Sections 5.1 and 5.2, we consider this problem for overdamped particles in linear and quadratic potentials, respectively. The stationary solution of the generalized Fokker-Planck equation (18) in the case of multiplicative Lévy stable noise and a tailored potential is presented in Section 5.3.

\subsection{Linear potential}

In this case $U(x, t)=U(x)=-f_{0} x\left(f_{0}\right.$ is a constant force acting on a particle) and, since $\mathcal{F}\{\partial f(x, t) P(x, t) / \partial x\}=$ $i k f_{0} P_{k}(t)$, equation (12) becomes

$$
\frac{\partial}{\partial t} P_{k}(t)=\left(-i k f_{0}+\phi_{k}\right) P_{k}(t) .
$$

The solution of this equation, satisfying the initial condition $P_{k}(0)=1$ that follows from the initial condition $P(x, 0)=\delta(x)$, is given by

$$
P_{k}(t)=\exp \left(-i k f_{0} t+\phi_{k} t\right) .
$$

Therefore, using the relation $S_{k}=e^{\phi_{k}}$, we can write the solution of the generalized Fokker-Planck equation (20) as

$$
P(x, t)=\mathcal{F}^{-1}\left\{S_{k}^{t} e^{-i k t f_{0}}\right\} .
$$


In order to study how the behavior of free particles, when $f_{0}=0$, depends on the character of the noise, we calculate the second moment $\left\langle x^{2}(t)\right\rangle=\int_{-\infty}^{\infty} d x x^{2} P(x, t)$ of the particle coordinate $x(t)$. Using the solution (51) and the integral formula $\delta^{\prime \prime}(k)=-(1 / 2 \pi) \int_{-\infty}^{\infty} d x x^{2} e^{i k x}$, we obtain

$$
\left\langle x^{2}(t)\right\rangle=-\left.\frac{d^{2}}{d k^{2}} S_{k}^{t}\right|_{k=0}
$$

Since in the case of free particles $x(t)=\eta(t)$, we have $S_{k}=$ $P_{k}(1)$, and the normalization of $P(x, t)$ yields $S_{0}=1$. Moreover, if the first moment of $x(t)$ exists, it is assumed to be zero, i.e., $d S_{k} /\left.d k\right|_{k=0}=0$. With these conditions, formula (52) reduces to

$$
\left\langle x^{2}(t)\right\rangle=\left\langle x^{2}(1)\right\rangle t
$$

If the term $\left\langle x^{2}(1)\right\rangle$, which can be represented as $\left\langle x^{2}(1)\right\rangle=$ $\lim _{\tau \rightarrow 0}\left\langle\left[\sum_{j=0}^{[1 / \tau]-1} \delta \eta(j \tau)\right]^{2}\right\rangle=\lim _{\tau \rightarrow 0}\left\langle\delta \eta^{2}(0)\right\rangle / \tau$ with $\left\langle\delta \eta^{2}(0)\right\rangle=\left\langle[\eta(\tau)-\eta(0)]^{2}\right\rangle=\int_{-\infty}^{\infty} d y y^{2} p(y, \tau)$, exists, i.e., $\left\langle\delta \eta^{2}(0)\right\rangle=O(\tau)$, then the noise is white and leads to normal diffusion of particles. In particular, $\left\langle x^{2}(t)\right\rangle=2 D t$ for Gaussian white noise, and $\left\langle x^{2}(t)\right\rangle=\lambda t \int_{-\infty}^{\infty} d y y^{2} q(y)$ for Poisson white noise. If the transition probability density $p(\Delta \eta, \tau)$ has heavy tails, i.e., $\left\langle\delta \eta^{2}(0)\right\rangle=\infty$, then $P(x, t)$ evolves in such a way that $\left\langle x^{2}(t)\right\rangle$ does not exist. Such behavior is displayed, e.g., by the probability density $P(x, t)=\mathcal{F}^{-1}\left\{S_{k}^{t}(\alpha, \beta, \gamma)\right\}$ of free particles driven by Lévy stable noise with $\alpha \in(0,2)$ [9]. While correlated noise induces a variety of different diffusion regimes of free particles [32], noise resulting from the noise generating process can only give rise to normal diffusive behavior (if $\left\langle\delta \eta^{2}(0)\right\rangle<\infty$ ) or to non-diffusive behavior, characterized by a probability density $P(x, t)$ with infinite second moment (if $\left\langle\delta \eta^{2}(0)\right\rangle=\infty$ ).

\subsection{Quadratic potential}

In the case of a quadratic potential, where $U(x, t)=$ $U(x)=b x^{2} / 2(b>0)$, equation (19) takes the form

$$
\frac{\partial}{\partial t} P_{k}(t)+b k \frac{\partial}{\partial k} P_{k}(t)=P_{k}(t) \phi_{k}
$$

Its general solution can be obtained via the method of characteristics [33], for example, and reads

$$
P_{k}(t)=\exp \left(\frac{1}{b} \int_{0}^{k} d z \frac{\phi_{z}}{z}+c_{1}\right) \Psi\left(\frac{1}{b} \ln |k|+c_{2}-t\right)
$$

where $\Psi(x)$ is an arbitrary function, and $c_{1}$ and $c_{2}$ are constants of integration. Since $P_{k}(0)=1$, the solution (55) yields

$$
\Psi\left(\frac{1}{b} \ln |k|+c_{2}\right)=\exp \left(-\frac{1}{b} \int_{0}^{k} d z \frac{\phi_{z}}{z}-c_{1}\right) .
$$

Replacing $k$ by $k e^{-b t}$ in this relation, we obtain

$$
\begin{aligned}
\Psi\left(\frac{1}{b} \ln |k|+c_{2}-t\right) & =\exp \left(-\frac{1}{b} \int_{0}^{k e^{-b t}} d z \frac{\phi_{z}}{z}-c_{1}\right) \\
& =\exp \left(-\frac{1}{b} \int_{0}^{k} d z \frac{\phi_{z e^{-b t}}}{z}-c_{1}\right)
\end{aligned}
$$

and substituting this result into (55), we find that

$$
P_{k}(t)=\exp \left(-\frac{1}{b} \int_{0}^{k} d z \frac{\phi_{z e^{-b t}}-\phi_{z}}{z}\right) .
$$

Finally, given that $\phi_{k}=\ln S_{k}$, the time-dependent solution of equation $(20), P(x, t)=\mathcal{F}^{-1}\left\{P_{k}(t)\right\}$, can be represented in the form

$$
P(x, t)=\mathcal{F}^{-1}\left\{\exp \left(-\frac{1}{b} \int_{0}^{k} d z \frac{1}{z} \ln \frac{S_{z e^{-b t}}}{S_{z}}\right)\right\} .
$$

We note that $\ln \left(S_{z e^{-b t}} / S_{z}\right) \sim-b t z d \ln S_{z} / d z$ as $b \rightarrow 0$. Therefore

$$
\lim _{b \rightarrow 0} \frac{1}{b} \int_{0}^{k} d z \frac{1}{z} \ln \frac{S_{z e^{-b t}}}{S_{z}}=-\ln S_{k}^{t},
$$

and in the case of free particles, i.e, $b=0$, the solution (59) reduces to $P(x, t)=\mathcal{F}^{-1}\left\{S_{k}^{t}\right\}$. This result is confirmed by the solution (51) with $f_{0}=0$.

\subsubsection{Poisson white noise}

As a first application of the above results we consider Poisson white noise. In this case $\phi_{k}=-\lambda\left(1-q_{k}\right)$, and formula (58) yields

$$
P_{k}(t)=\exp \left(-\frac{\lambda}{b} \int_{0}^{k} d z \frac{q_{z e^{-b t}}-q_{z}}{z}\right) .
$$

Next we assume that the probability density $q(\Delta \eta)$ is exponential, i.e., $q(\Delta \eta)=(r / 2) e^{-r|\Delta \eta|}$ with $r>0$. This implies that $q_{k}=r^{2}\left(r^{2}+k^{2}\right)^{-1}$,

$$
\begin{aligned}
\int_{0}^{k} d z \frac{q_{z e^{-b t}-q_{z}}}{z} & =\int_{0}^{k} d z \frac{r^{2}\left(1-e^{-2 b t}\right) z}{\left(r^{2}+z^{2}\right)\left(r^{2}+z^{2} e^{-2 b t}\right)} \\
& =\frac{1}{2} \ln \frac{r^{2}+k^{2}}{r^{2}+k^{2} e^{-2 b t}}
\end{aligned}
$$

and formula (61) takes the form

$$
P_{k}(t)=\left(\frac{r^{2}+k^{2} e^{-2 b t}}{r^{2}+k^{2}}\right)^{\frac{\lambda}{2 b}} .
$$

For $t \rightarrow \infty$ the time-dependent solution $P(x, t)=$ $\mathcal{F}^{-1}\left\{P_{k}(t)\right\}$ of the generalized Fokker-Planck equation $(20)$ tends to the stationary solution $P_{\mathrm{st}}(x)=$ $\mathcal{F}^{-1}\left\{P_{k}(\infty)\right\}$. Using the relation [34]

$\int_{0}^{\infty} d k \frac{\cos k x}{\left(r^{2}+k^{2}\right)^{s}}=\frac{\sqrt{\pi}}{\Gamma(s)}\left(\frac{|x|}{2 r}\right)^{s-1 / 2} K_{s-1 / 2}(r|x|)$, 
where Re $\mathrm{s}>0$ and $K_{l}(x)$ is the modified Bessel function of the third kind (or Macdonald function) [35], we obtain

$$
P_{\mathrm{st}}(x)=\sqrt{\frac{2}{\pi}} \frac{r(r|x|)^{s-1 / 2}}{2^{s} \Gamma(s)} K_{s-1 / 2}(r|x|)
$$

with $s=\lambda /(2 b)$. This is the so-called $K$-distribution, which is one of the basic distributions describing the statistical properties of scattered waves [36]. It is interesting to note that for $s \leq 1 / 2$ the stationary probability density exhibits singular behavior near the bottom $(x=0)$ of the potential well: $P_{\text {st }}(x) \propto 1 /|x|^{1-2 s}$ if $0<s<1 / 2$, and $P_{\text {st }}(x) \propto-\ln |x|$ if $s=1 / 2$.

\subsubsection{Lévy stable noise}

In this case $S_{k}=S_{k}(\alpha, \beta, \gamma)$, and the characteristic function (33) implies that

$$
\ln \frac{S_{z e^{-b t}}}{S_{z}}=\gamma\left(1-e^{-\alpha b t}\right)|z|^{\alpha}\left(1+i \beta \operatorname{sgn}(z) \tan \frac{\pi \alpha}{2}\right) .
$$

Using this result and the integral formula

$$
\int_{0}^{k} d z \frac{|z|^{\alpha}}{z}\left[\begin{array}{c}
\operatorname{sgn} z \\
1
\end{array}\right]=\frac{|k|^{\alpha}}{\alpha}\left[\begin{array}{c}
\operatorname{sgn} k \\
1
\end{array}\right],
$$

we find that

$$
\begin{aligned}
\int_{0}^{k} d z \frac{1}{z} \ln \frac{S_{z e^{-b t}}}{S_{z}}= & \frac{\gamma}{\alpha}\left(1-e^{-\alpha b t}\right)|k|^{\alpha} \\
& \times\left(1+i \beta \operatorname{sgn}(k) \tan \frac{\pi \alpha}{2}\right),
\end{aligned}
$$

and the solution (59) reads

$$
P(x, t)=\mathcal{F}^{-1}\left\{S_{k}\left(\alpha, \beta, \gamma \frac{1-e^{-\alpha b t}}{\alpha b}\right)\right\} .
$$

We note that special cases of this solution were known previously. Specifically, the time-dependent solution for symmetric Lévy stable noise $(\beta=0)$ was derived in reference [9], and the steady-state solution for asymmetric Lévy stable noise in reference [37].

\subsection{Tailored potential}

As a third example we derive the stationary probability density function $P_{\text {st }}(x)$ for overdamped particles interacting with a tailored potential

$$
U(x)=c \int_{0}^{x} d y y g^{\alpha}(y)
$$

$(c>0)$ and driven by multiplicative Lévy stable noise with $g(x, t)=g(x)>0$. In this case $f(x, t)=-c x g^{\alpha}(x)$, and equation $(35)$ reduces to

$$
c \frac{d}{d x} x G(x)+\mathcal{F}^{-1}\left\{G_{k} \ln S_{k}(\alpha, \beta, \gamma)\right\}=0,
$$

where $G(x)=g^{\alpha}(x) P_{\text {st }}(x)$. By applying the Fourier transform to equation (71), we arrive at the ordinary differential equation

$$
-c k \frac{d}{d k} G_{k}+G_{k} \ln S_{k}(\alpha, \beta, \gamma)=0,
$$

whose integration yields

$$
\ln \frac{G_{k}}{G_{0}}=\frac{1}{c} \int_{0}^{k} d z \frac{1}{z} \ln S_{z}(\alpha, \beta, \gamma) .
$$

Using the definition (33) and the integral formula (67), we obtain

$$
G_{k}=G_{0} S_{k}(\alpha, \beta, \gamma / \alpha c)
$$

and so

$$
P_{\text {st }}(x)=G_{0} g^{-\alpha}(x) \mathcal{F}^{-1}\left\{S_{k}(\alpha, \beta, \gamma / \alpha c)\right\} .
$$

To eliminate $G_{0}=\left\langle g^{\alpha}(x(t))\right\rangle$ from the solution (75), we use the normalization condition $\int_{-\infty}^{\infty} d x P_{\text {st }}(x)=1$, which yields the desired probability density

$$
P_{\mathrm{st}}(x)=\frac{g^{-\alpha}(x) \mathcal{F}^{-1}\left\{S_{k}(\alpha, \beta, \gamma / \alpha c)\right\}}{\int_{-\infty}^{\infty} d x g^{-\alpha}(x) \mathcal{F}^{-1}\left\{S_{k}(\alpha, \beta, \gamma / \alpha c)\right\}} .
$$

To the best of our knowledge, the above result is the first stationary solution of the fractional Fokker-Planck equation (44), i.e., the generalized Fokker-Planck equation (18) associated with the Langevin equation (1) driven by multiplicative Lévy stable noise.

\section{Conclusions}

We have derived a new form of the generalized Fokker-Planck equation associated with the Langevin equation for overdamped particles driven by multiplicative noise which results from the noise generating process whose independent increments have an arbitrary distribution. The main advantage of this generalized Fokker-Planck equation is that it accounts for the noise action in a unified way, namely through the characteristic function of the noise generating process at dimensionless time $t=1$. Since the characteristic function is completely described by the transition probability density of the generating process, it is this density which ultimately determines the term in the generalized Fokker-Planck equation that describes the effect of the noise on the dynamics of the system. We have explicitly demonstrated this fact for various noises, including the Poisson white noise and the Lévy stable noise.

More importantly, we have solved the generalized Fokker-Planck equation in the cases of linear and quadratic potentials driven by an arbitrary additive noise. A remarkable feature of these analytical solutions is that they give an opportunity to examine the effects of different noises on the same system. As an illustration, we have derived and analyzed in detail the time-dependent and stationary solutions that correspond to the Poisson white and 
Lévy stable noises. Moreover, we have also presented the analytical solution of the fractional Fokker-Planck equation that describes the stationary distribution of overdamped particles in a specific potential driven by multiplicative Lévy stable noise.

S.I.D. acknowledges the support of the EU through Contract No. MIF1-CT-2006-021533, and P.H. acknowledges financial support by the Deutsche Forschungsgemeinschaft via the Collaborative Research Centre SFB-486, Project No. A 10, and by the German Excellence Cluster "Nanosystems Initiative Munich" (NIM).

\section{References}

1. P. Langevin, C. R. Acad. Sci. 146, 530 (1908)

2. W.T. Coffey, Yu.P. Kalmykov, J.T. Waldron, The Langevin Equation, 2nd edn. (World Scientific, Singapore, 2004)

3. N.G. Van Kampen, Stochastic Processes in Physics and Chemistry (North-Holland, Amsterdam, 1992)

4. W. Horsthemke, R. Lefever, Noise-Induced Transitions (Springer-Verlag, Berlin, 1984)

5. P. Hänggi, H. Thomas, Phys. Rep. 88, 207 (1982)

6. H. Risken, The Fokker-Planck Equation, 2nd edn. (Springer-Verlag, Berlin, 1989)

7. K. Sato, Lévy processes and infinitely divisible distributions, (Cambridge University Press, Cambridge, 1999)

8. C.W. Gardiner, Handbook of Stochastic Methods, 2nd edn. (Springer-Verlag, Berlin, 1990)

9. S. Jespersen, R. Metzler, H.C. Fogedby, Phys. Rev. E 59, 2736 (1999)

10. P.D. Ditlevsen, Phys. Rev. E 60, 172 (1999)

11. R. Metzler, J. Klafter, Phys. Rep. 339, 1 (2000)

12. V.V. Yanovsky, A.V. Chechkin, D. Schertzer, A.V. Tur, Physica A 282, 13 (2000)

13. D. Brockmann, I.M. Sokolov, Chem. Phys. 284, 409 (2002)

14. A.V. Chechkin, V.Y. Gonchar, J. Klafter, R. Metzler, Adv. Chem. Phys. 133, 439 (2006)
15. S.I. Denisov, W. Horsthemke, P. Hänggi, Phys. Rev. E 77, $061112(2008)$

16. P. Hänggi, P. Jung, Adv. Chem. Phys. 89, 239 (1995)

17. I.I. Gikhman, A.V. Skorokhod, The Theory of Stochastic Processes (Springer, Berlin, 2004), Vol. 1

18. K. Ito, Nagoya Math. J. 1, 35 (1950)

19. S.I. Denisov, A.N. Vitrenko, W. Horsthemke, Phys. Rev. E 68, 046132 (2003)

20. I. Eliazar, J. Klafter, J. Stat. Phys. 111, 739 (2003)

21. I. Eliazar, J. Klafter, J. Stat. Phys. 119, 165 (2005)

22. A. Dubkov, B. Spagnolo, Fluct. Noise Lett. 5, L267 (2005)

23. A. Dubkov, B. Spagnolo, V.V. Uchaikin, Int. J. Bifurcat. Chaos 18, 2649 (2008)

24. W. Feller, An Introduction to Probability Theory and its Applications, 2nd edn. (Wiley, New York, 1971), Vol. 2

25. P. Hänggi, Z. Phys. B 30, 85 (1978)

26. P. Hänggi, Z. Phys. B 36, 271 (1980)

27. N.G. van Kampen, Physica A 102, 489 (1980)

28. P. Hänggi, Z. Phys. B 31, 407 (1978)

29. B.V. Gnedenko, A.N. Kolmogorov, Limit Distributions for Sums of Independent Random Variables (Addison-Wesley, Cambridge, MA, 1954)

30. V.M. Zolotarev, One-Dimensional Stable Distributions (American Mathematical Society, Providence, 1986)

31. S.G. Samko, A.A. Kilbas, O.I. Marichev, Fractional Integrals and Derivatives: Theory and Applications (Gordon \& Breach, New York, 1993)

32. S.I. Denisov, W. Horsthemke, Phys. Rev. E 62, 7729 (2000)

33. A.D. Polyanin, V.F. Zaitsev, A. Moussiaux, Handbook of First-Order Partial Differential Equations (Taylor \& Francis, London, 2002)

34. A.P. Prudnikov, Yu.A. Brychkov, O.I. Marichev, Integrals and Series (Gordon \& Breach, New York, 1986), Vol. 1, Eq. (2.5.6.4)

35. H. Bateman, A. Erdélyi, Higher Transcendental Functions (McGraw-Hill, New York, 1953), Vol. 2

36. E. Jakeman, R.J.A. Tough, Adv. Phys. 37, 471 (1988)

37. B. Dybiec, E. Gudowska-Nowak, I.M. Sokolov, Phys. Rev. E 76, 041122 (2007) 